\title{
A Prospective Observational Study of Ventilator-Associated Pneumonia in a Tertiary Care Centre, KGH, Visakhapatnam
}

\author{
Satya Kumari Kunche ${ }^{1}$, Sujatha Devi Rapaka² \\ 1, 2 Department of Paediatrics, Andhra Medical College, NTR University of Health Sciences, \\ Visakhapatnam, Andhra Pradesh, India.
}

\section{ABSTRACT}

\section{BACKGROUND}

Ventilator-associated pneumonia (VAP) is a nosocomial pneumonia ${ }^{1}$ in mechanically ventilated children that develops after $48 \mathrm{hrs}$ of initiation. It is one of the leading causes of morbidity and mortality among hospital-acquired infections. ${ }^{2,3}$ VAP complicates $8-28 \%$ of the children receiving mechanical ventilation. The incidence of VAP is estimated to be 3 to $10 \%$ of the ventilated PICU children in developed countries. Studies from India have shown a contrastingly high range of VAP rates of $6 \%$ to $46 \%$. The mortality rate with VAP considerably increased, varying from 24 to $50 \%$ and can reach as high as $76 \%$ when high-risk pathogens cause lung infection. ${ }^{4}$ Several risk factors predispose to VAP development during mechanical ventilation, as shown in different studies. Most of the risk factors are preventable, which can reduce the incidence of VAP when appropriately managed. As there is an increased use of mechanical ventilation in developing countries like India because of delays in reaching the hospital, the condition of the child is critical. The poor living conditions of the children who come to the tertiary care centres from remote rural areas often present with poor general conditions. We wanted to know about the risk factors, organisms causing VAP, their antibiotic sensitivity, and for optimal VAP management to reduce health care costs, guide antibiotic therapy, and reduce the morbidity and mortality associated with the VAP.

\section{METHODS}

A prospective observational study was done at PICU, King George Hospital, Visakhapatnam from December 2018 to July 2020 for a period of 20 months on children aged $>1$ month to 12 years, who were kept more for than 48 hours in PICU. The sample size was 126 .

\section{RESULTS}

All PICU children between theage group of $>1$ month and $<12$ years who received mechanical ventilation (MV) for $>48$ hours were consecutively enrolled in the study. A total of 126 children were enrolled during the study period.

\section{CONCLUSIONS}

VAP is an important nosocomial infection in PICU with an incidence of $26 \%$ and was associated with a high mortality rate of $38.5 \%$. Gram-negative bacilli (acinetobacter and klebsiella) were the important causative agents that were nearly resistant to commonly used antibiotics.

\section{KEY WORDS}

ARDS, BAL, CPIS, ETA, VAP, MDR, MASA. HF, PAWP.
Corresponding Author: Dr. Satya Kumari Kunche, Assistant Professor, Department of Paediatrics, Andhra Medical College, NTR University of Health Sciences, Visakhapatnam, Andhra Pradesh, India. E-mail:drmvsvalluri@gmail.com

DOI: $10.14260 /$ jemds/2022/67

How to Cite This Article:

Kunche SK, Rapaka SD. A prospective observational study of ventilator associated pneumonia in a tertiary care centre, KGH, Visakhapatnam. J Evolution Med Dent Sci 2022;11(02):347-351, DOI: 10.14260/jemds/2022/67

Submission 24-12-2021, Peer Review 31-12-2021, Acceptance 29-01-2022, Published 04-02-2022.

Copyright (C) 2022 Satya Kumari Kunche et al. This is an open access article distributed under Creative Commons Attribution License [Attribution 4.0 International (CC BY 4.0)] 


\section{BACKGROUND}

Ventilator-associated Pneumonia (VAP) is nosocomial pneumonia ${ }^{1}$ in (invasive) mechanically ventilated children. ${ }^{4}$ It is one of the leading causes of morbidity and mortality in hospital acquired ${ }^{2,3}$ pneumonia. It develops in more than 48 hours after the initiation of a mechanical ventilator (MV). ${ }^{5}$ Several risk factors predispose to VAP development. The use of appropriate antibiotics ${ }^{6}$ directed towards the most prevalent organism improves the cure rate and survival and reduces the emergence of resistant strains. The incidence of VAP in the majority of the studies, varied between 9 and 46 $\%$. The risk of development of VAP was $3 \%$ per day in the first 5 days, $2 \%$ per day in 5 to 10 days and $1 \%$ per day thereafter in mechanically ventilated children. The intubation process itself increases the risk of infection; hence to prevent VAP in children with acute respiratory failure, non-invasive methods are helpful.

\section{Mortality}

Crude ICU mortality rates of 16 to $57 \%$ have been reported for VAP. In VAP, there is a 2-10 fold higher risk of death compared with children without pneumonia. The attributable mortality of VAP has been estimated to be between 33 and 50 $\%$. Increased mortality rates in VAP were associated with bacteraemia, especially gram-negative with Pseudomonas aeruginosa or acinetobacter species and ineffective antibiotic therapy due to multidrug-resistant pathogens. Few studies with similar methodology failed to identify attributable mortality, suggesting a variable outcome, according to the severity of underlying medical conditions. ${ }^{2}$

\section{Objectives}

1. To determine the incidence and outcome of VAP in a tertiary care centre, like King George Hospital (KGH).

2. To identify the probable risk factors for VAP.

3. To identify the common pathogenic bacteria causing VAP.

\section{METHODS}

This is a prospective observational study that was done on children aged $>1$ month to 12 years who were kept $>48$ hours in PICU, King George Hospital, Visakhapatnam. It was done for a period of 20 months from December 2018 to July 2020. 126 children were enrolled for the study.

\section{Inclusion Criteria}

- $\quad$ Age $>1$ month to 12 years.

- $\quad$ All PICU children who were mechanically ventilated for more than 48 hours during the study period.

\section{Exclusion Criteria}

Age less than 1 month and more than 12 years, children with pre-existing radiological infiltrate at the beginning of ventilation, children with pre-existing pulmonary infection at the beginning of ventilation.

\section{Diagnosis of VAP \\ Definite $V A P^{7,8}$}

A child who fulfils the case definition for hospital-acquired pneumonia and has one of the following:

The same pathogen is isolated from bronchial secretions or $\mathrm{BAL}^{9}$ and blood. The pathogen either bacteria or virus isolated from lung biopsy, or positive growth in culture of pleural fluid, or histopathology examination with evidence of pneumonia manifested as abscess formation, positive culture of the lung parenchyma or fungal hyphae.

Pathogen like bacteria or virus isolated from BAL (bacteria $\geq 104 \mathrm{CFU} / \mathrm{ml}$ ), or $\geq 5 \%$ of BAL-obtained cells contain intracellular bacteria on a direct microscopic exam or protected brush with a threshold of $\geq 104 \mathrm{CFU} / \mathrm{ml}$, or positive exams for particular microorganisms (legionella, aspergillus, mycobacteria, mycoplasma, $P$. jirovercii)

VAP is considered in mechanically ventilated children for more than 48 hours with a clinical pulmonary infection score (CPIS) of 6 or more. A cut off of 96 hours of mechanical ventilation is used to distinguish the early onset of VAP from late-onset VAP. Possible VAP: A child who fulfils the case definitions for hospital-acquired pneumonia with nonquantitative lower respiratory tract specimen culture or no positive microbiology.

Clinical, radiological, microbiological diagnosis of VAP based on clinical pulmonary infection score (CPIS) system. ${ }^{10}$ Probable VAP: A child who fulfils the case definitions for hospital-acquired pneumonia and has one of the following: 15 - Pathogen isolated from BAL ${ }^{11}$ (bacteria < $104 \mathrm{CFU} / \mathrm{ml}$ ) Pathogen or virus isolated from bronchial secretions, or quantitative culture of lower respiratory tract specimen (endotracheal aspirate) with a threshold of bacteria $\geq 106$ $\mathrm{CFU} / \mathrm{ml}$.

\section{CPIS Score ARDS 10}

ARDS: Acute respiratory distress syndrome; HF: heart failure; PAWP: pulmonary artery wedge pressure.

\begin{tabular}{|ccr|}
\hline & Parameter & Points \\
\hline Temperature & $>36.5$ and $<38.4$ & 0 \\
(Degree Celsius) & $>36.5$ and $<38.9$ & 1 \\
Blood Leukocyte & $>39.0$ and $<36.0$ & 2 \\
Count/Microliter & $>4000$ and $<11000$ & 0 \\
Tracheal Secretions & No00 and 11000 (With band forms $>5 \%)$ & $1(+1)$ \\
& Non-Purulent Secretions Present & 0 \\
Oxygenation & Purulent Secretions Present & 1 \\
& PaO2/FiO2 in mm of Hg & 2 \\
Pulmonary & $>240$ or ARDS & 0 \\
Radiography & Radiographic Progression (CCF and ARDS excluded) & 2 \\
Pathogenic Bacteria & No Infiltrate & 0 \\
Cultured from & Moderate / Heavy Growth (with same growth on & 0 \\
Tracheal Aspirates & grams stain) & $1(+1)$ \\
\hline & & \\
\hline
\end{tabular}

All PICU children fulfilling inclusion criteria were enrolled in the study. After taking the informed written consent from the parent or guardian, the relevant information from the history, physical examination investigation findings were recorded in a well-designed proforma.

\section{Clinical Criteria}

The standard diagnostic criteria for two of the following three findings: 
- Fever, leukocytosis, and purulent tracheal secretions. usually with

- Abnormal chest radiographic studies. Pugin et al. developed a composite clinical score, called the clinical pulmonary infection score (CPIS) 6.10

The score varied from $0-12$, a CPIS of $>6$ has a sensitivity of $93 \%$ and specificity of $100 \%$.

ARDS is defined as $\mathrm{PaO} 2 / \mathrm{FiO} 238.5^{\circ} \mathrm{C}$. Leukocytosis is defined as a $25 \%$ increase in circulating leukocytes from baseline and a leucocyte count of $>10,000 / \mathrm{mm} \mathrm{3}$. Tracheal aspirates are considered purulent if a Gram's stain showed > 25 neutrophils per high-power field. Tracheal aspirates will be sent for bacterial culture and sensitivity.

\section{Microbiological Diagnosis}

Specimens can be obtained by:

1. Non-invasive techniques: Blood and endotracheal aspirates

2. Invasive techniques

a. Bronchoscopic technique - Fiberoptic bronchoscopy BAL (Bronchoalveolar lavage) - PSB (Protected specimen brush)

b. Non-bronchoscopic technique - Blinded invasive methods - Blinded bronchial sampling -Mini BAL (Bronchoalveolar lavage) - Blinded PSB (Protected specimen brush).

The emergence of selected multidrug-resistant bacteria rates of VAP due to MDR pathogens has increased dramatically in hospitalized children, especially in intensive care and transplant children. Pseudomonas aeruginosa, the most common MDR gram-negative bacteria causing VAP, has intrinsic resistance to many antimicrobial agents. This resistance is mediated by multiple efflux pumps, which may be up-regulated by mutation. Resistance to piperacillin, ceftazidime, cefepime, imipenem and meropenem, aminoglycosides, or fluoroquinolones is increasing.

Klebsiella species are resistant to ampicillin and other aminopenicillins and can acquire resistance to cephalosporins and aztreonam by the production of extended-spectrum $\beta$ lactamases (ESBL).

\section{Radiologic Diagnosis}

The initial diagnosis of VAP is based on clinical suspicion and the presence of new or progressive radiographic infiltrates.

The presence of alveolar infiltrates, air bronchogram (sensitivity of 58 to $83 \%$ ) and new or worsening infiltrates (sensitivity of 50-78 \%) diagnoses VAP.

The presence of any one radiographic sign does not significantly increase the likelihood of VAP, because other causes of radiographic abnormalities occur in ventilator assisted children. Endotracheal aspiration, ARDS, pulmonary oedema and atelectasis

\section{Data Collection}

All PICU children fulfilling inclusion criteria were enrolled in the study. After taking the informed written consent from the parent or guardian, the relevant information from the history, physical examination and investigation findings were recorded in a well-designed proforma.

\section{RESULTS}

\begin{tabular}{|ccc|}
\hline \multicolumn{2}{|c|}{ CPIS Score } & $\%$ \\
\hline Score & $7-8$ & 9 \\
Score & $9-10$ & 24 \\
Score & $11-12$ & 3 \\
\hline Table 1. Distribution of CPIS Score in VAP Children Total $(N=36)$ \\
\hline
\end{tabular}
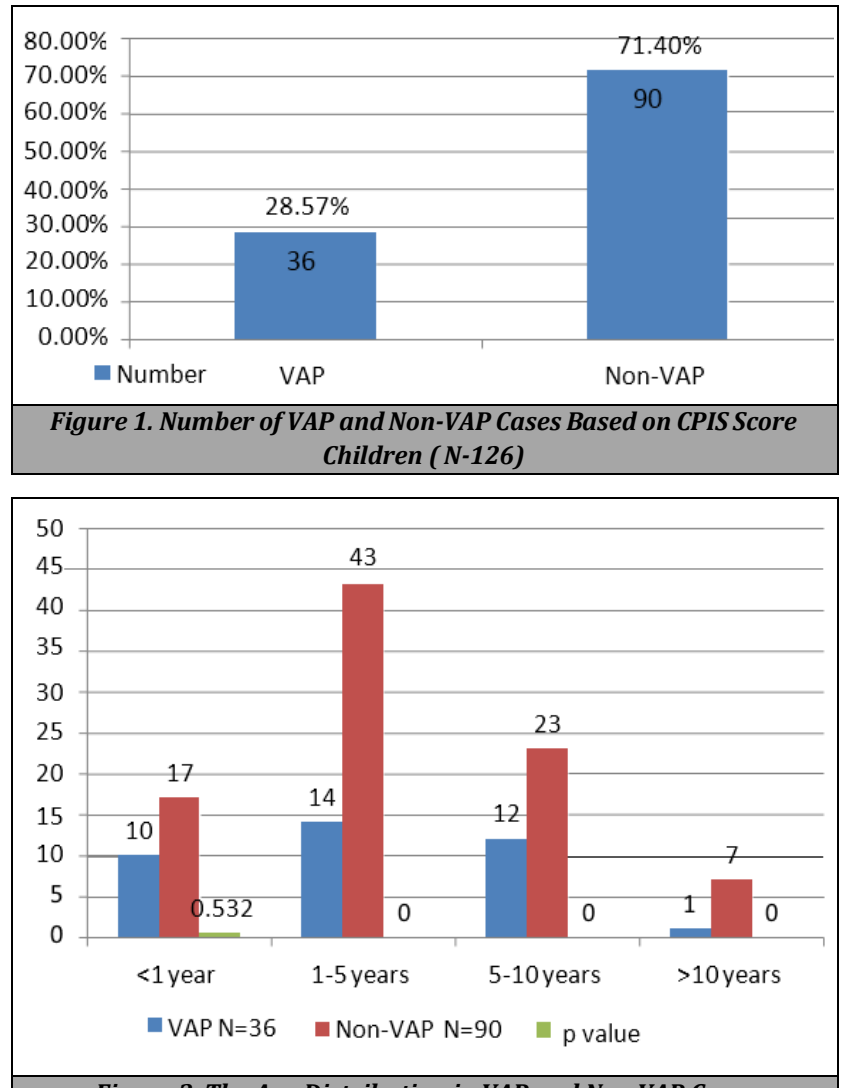

Figure 3. The Age Distribution in VAP and Non VAP Cases

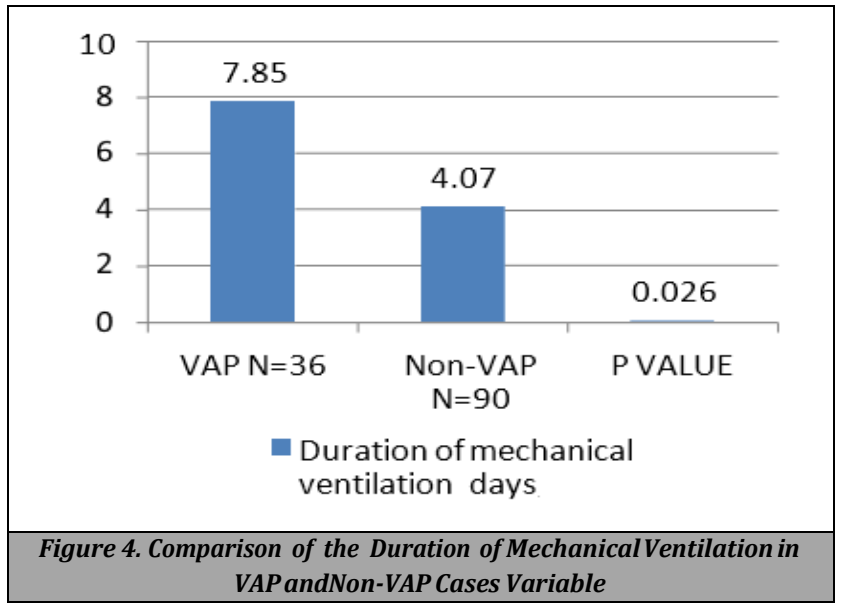




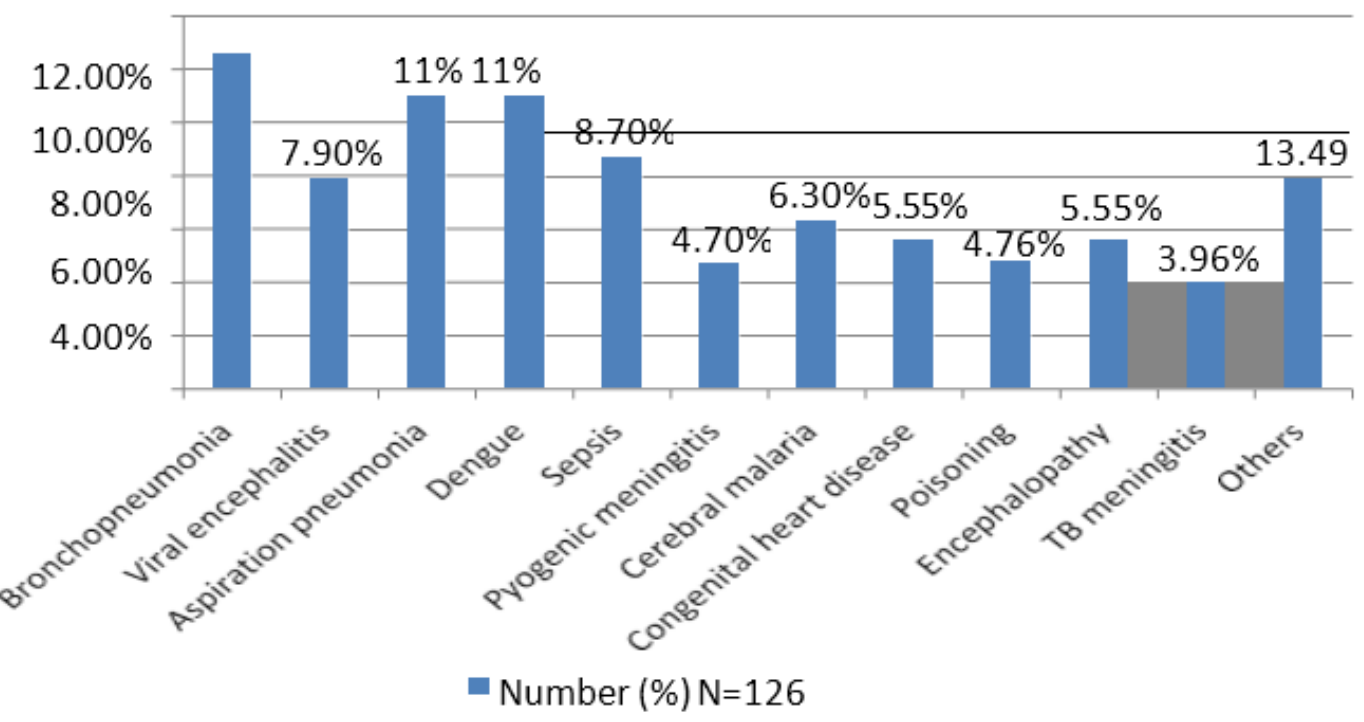

Figure 2. Frequency Distribution of the Primary Diagnoses of Cases Receiving Mechanical Ventilation.

\begin{tabular}{|cccc|}
\hline Primary Diagnosis & Total N=126 & VAP N=36 & Non-VAPN=90 \\
CNS & 33 & 9 & 24 \\
Bronchopneumonia & 48 & 9 & 39 \\
Dengue & 12 & 6 & 6 \\
Sepsis & 24 & 8 & 16 \\
CVS & 6 & 2 & 4 \\
GIT & 3 & 2 & 1 \\
NMS & $2(2 \%)$ & $1(3.8 \%)$ & $1(1.3 \%)$ \\
Snake poisoning & $2(2 \%)$ & $1(3.8 \%)$ & $1(1.3 \%)$ \\
OP poisoning & $2(2 \%)$ & 0 & $2(2.7 \%)$ \\
Hydrocarbon poisoning & $1(1 \%)$ & 0 & $1(1.3 \%)$ \\
\hline Table 2. Comparison of Primary Diagnosis of Cases Receiving \\
Mechanical Ventilation (MV) betweenVAP and Non-VAP Cases \\
\multicolumn{4}{c}{ System Involved } \\
\hline
\end{tabular}

\section{DISCUSSION}

Mechanical Ventilator (MV) is an essential component of modern ICU care and it is associated with a considerable risk of ventilator-associated pneumonia (VAP). Proper recognition of high-risk children and potentially modifiable risk factors may outline preventive measures and institutional strategies to reduce the infection. VAP may be polymicrobial ${ }^{12}$ and are rarely due to viral or fungal pathogens in immunocompetent hosts. Common pathogens include aerobic gram-negative organisms, such as P. aeruginosa, E. coli, klebsiella and acinetobacter species. Infections due to the gram-positive organism, such as Staphylococcus aureus, particularly Methicillin-resistant $S$. aureus (MRSA), have been rapidly emerging. Pneumonia due to $S$. aureus ${ }^{13}$ is more common in children with diabetes mellitus, head trauma, and those hospitalized in ICUs.

The present study evaluated 126 children aged 1-12 years who received mechanical ventilation (MV) in paediatric intensive care unit (PICU) in a tertiary care centre, $\mathrm{KGH}$, Visakhapatnam. In this study, the incidence and outcome of ventilator-associated pneumonia (VAP), the risk factors for VAP and the microbiological spectrum of VAP were evaluated. The incidence of VAP in the present study is comparable to the incidence of VAP in the study done by Vedavathy $\mathrm{S}$ et al. ${ }^{14}$ from IGICH.
Bengaluru was $22.6 \%$ and comparable to the incidence of VAP in the study done by Patra PK 15 from PGI, Chandigarh which was $30.5 \%$. There was a wide range of variations in the reported incidence rates of VAP at various centres, the present study correlates with various Indian studies. The difference in incidence rates could be mainly due to different criteria used for the diagnosis of VAP, sample size and the underlying disease state requiring ventilator support.

Early-onset VAP, which occurs within the first 4 days of mechanical ventilation, usually carries a better prognosis and is more likely caused by antibiotic sensitive bacteria. Lateonset VAP (more than 5 days) is more likely caused by multidrug-resistant (MDR) pathogens and is associated with increased child mortality and morbidity. In the present study among VAP cases, $46.1 \%$ of cases had early VAP and $53.8 \%$ cases had late VAP. We observed that $82.35 \%$ of children with a history of 3-4 intubations and $100 \%$ of children with $\geq$ 5 intubations had developed VAP in our study.

Important risk factor for the development of VAP is more the number of intubations, more the number of VAP cases. In the present study, VAP was significantly associated (P-value 0.00 ) with a higher number of intubations. Re-intubation is the independent risk factor of VAP. ${ }^{16,17}$ Nemat $^{18}$ B, Habibi P. Does Re-intubation Increase Risk of Ventilator-Associated Pneumonia (VAP) in Paediatric Intensive Care Unit Children? Int J Pediatr. 2015 January; 3(1-1):411-15

We observed that the presence of nasogastric tube and early enteral feeding was significantly associated with the occurrence of VAP (P-value 0.00), it is because aspiration of stomach contents into the lungs might be the reason for the development of aspiration pneumonia. Out of 18 children with a nasogastric tube, 13 children (72.22 \%) developed VAP in our study. Presence of nasogastric tube in situ is a riskfactor for the development of VAP which is comparable with the present study. In the microbiological spectrum of VAP, the organisms isolated from the endotracheal aspirates of the current study participants with VAP were acinetobacter species, Pseudomonas aeruginosa, klebsiella pneumonia, Staphylococcus aureus and Enterobacter.12,19 Acinetobacter $(38.46 \%)$ was the most common organism. ${ }^{20}$ 
There are other procedures to obtain material for the microbiological study; the major limitation of the present study was the ability to use newer techniques (such as Bronchoalveolar Lavage or Protected specimen brush technique) due to resource constraints and use of less specific clinical criteria for diagnosis of VAP.

\section{CONCLUSIONS}

The present prospective study was done at a tertiary care hospital in children aged 1 month -12 years on mechanical ventilation for more than 48 hours being admitted in PICU, $\mathrm{KGH}$, Visakhapatnam. A total of 126 children $(76$ males and 50 females) were enrolled in the study. The mean duration of mechanical ventilation was $5.05 \pm 2.30$ days and the mean duration of ICU stay was 7.34 2.93 days. The incidence of VAP based on CPIS score > 6 was $26 \%$. The majority of the children $(84.6 \%)$ had a CPIS score of $>8$. Age at presentation and gender were not significantly associated with the occurrence of VAP. The presence of uncuffed endotracheal tube for intubation and history of multiple intubations was significantly associated with VAP. The presence of the central venous line and nasogastric tube, and increased duration of mechanical ventilator were also significantly associated with VAP. Acinetobacter species, Pseudomonas aeruginosa and Klebsiella pneumonia were the most common pathogens for causing VAP. The overall mortality rate of VAP in our study population was $38.5 \%$. Out of $10 \mathrm{VAP}$ deaths in the present study, $40 \%$ of VAP death cases were secondary to gramnegative infections with acinetobacter followed by klebsiella (30\%), pseudomonas (20\%) and Enterobacter.

\section{REFERENCES}

[1] Payal PM, Tanuja JB, Sandeep N, et al. A study on ventilator associated pneumonia in pediatric age gropu in a tertiary care hospital, Vadodara. National Journal of Medical Research 2012;2(3):318-21.

[2] Mahantesh S, Bhavana J, Basavaraj GV, et al. Associated pneumonia inpaediatric intensive care unit at the Indira Gandhi Institutue of child health. Indian Journal of Immunology and Respiratory Medicine 2017;2(2):36-41.

[3] Cook DJ, Walter SD, Cook RJ, et al. Incidence of and risk factors for ventilator-associated pneumonia in critically ill children. Ann Intern Med 1998;129(6):433-40.

[4] Vijay G, Kabra SK, Lodha R, et al. Ventilator-associated pneumonia in pediatric intensive care unit. Indian J Pediatr 2018;85(10):861-6.

[5] Katherason GS, Naing L, Jaalam K, et al. Ventilatorassociated nosocomial pneumonia in intensive care units in Malaysia. J Inf Dev Ctries 2009;3(9):704-10.
[6] Pugin J, Auckenthaler R, Mili N, et al. Diagnosis of ventilator associated pneumonia by bacteriologic analysis of bronchoscopic and non bronchoscopic "blind" bronchoalveolar lavage fluid. Am Rev Respir Dis 1991;143(5 Pt 1):1121-9.

[7] Papazian L, Thomas P, Garbe L, et al. Bronchoscopic or blind sampling techniques for the diagnosis of ventilatorassociated pneumonia. Am J Respir Crit Care Med 1995;152(6 Pt 1):1982-91.

[8] Klompas M, Magill S, Robicsek A, et al. Objective surveillance definitions for ventilator-associated pneumonia. Crit Care Med 2012;40(12):3154-61.

[9] Galal YS, Youssef MRL, Ibrahiem SK. VentilatorAssociated Pneumonia: Incidence, risk factors and outcome in paediatric intensive care units at Cairo University Hospital. J Clin Diagn Res 2016;10(6):SC6-11.

[10] Rosbolt MB, Sterling ES, Fahy BG. The utility of the clinical pulmonary infection score. J Intensive Care Med 2009;24(1):26-34.

[11] Fagon JK, Chastre J, Domart Y, et al. Nosocomial pneumonia in children receiving continuous mechanical ventilation. Prospective analysis of 52 episodes with use of a protected specimen brush and quantitative culture techniques. Am Rev Respir Dis 1989;139(4):877-8.

[12] Rello J, Rue M, Jubert P, et al. Survival in children with nosocomial pneumonia: impact of the severity of illness and the etiologic agent. Crit Care Med 1997;25(11):1862-7.

[13] Rello J, Torres A, Ricart M, et al. Ventilator-associated pneumonia by Staphylococcus aureus. Comparison of methicillin-resistant and methicillin-sensitive episodes. Am J Respir Crit Care Med 1994;150(6 Pt 1):1545-9.

[14] Vedhavathy S, Sangamesh. Clinical study of ventilator associated pneumonia in a tertiary care centre. Int J Contemp Pediatr 2016;3(2):432-41.

[15] Patra PK, Jayashree M. Incidence, risk factors, outcome and microbiological profile ventilator associated pneumonia in PICU. Indian Pediatrics 2007;44:511-8.

[16] Elward AM, Warren DK, Fraser VJ. Ventilator-associated pneumonia in Pediatric Intensive Care Unit children: risk factors and outcomes. Pediatrics 2002;109(5):758-64.

[17] Amro K. Reintubation increases ventilator-associated pneumonia in pediatric intensive care unit children. Rawal Med J 2008;33(2):145-9.

[18] Nemat B, Habibi P. Does Re-intubation increased risk of ventilator associated pneumonia (VAP) in paediatric intensive care unit children? Int J Pediatr 2015;3(11):411-5.

[19] Rello J, Gallego M, Mariscal D, et al. The value of routine microbial investigation in ventilator-associated pneumonia. Am J Respir Crit Care Med 1997;156(1):196200.

[20] Balasubramanian P, Tullu MS. Study of ventilatorassociated pneumonia in a pediatric intensive care unit. Indian J Pediatr 2014;81(11):1182-6. 